\title{
Mapa conceptual en salud y propiedad intelectual en Centroamérica y la República Dominicana
}

\author{
Beatriz M. García Delgado, ${ }^{1}$ Ana Paula Silva ${ }^{\dagger}$ y Juana M. de Rodriguez ${ }^{3}$
}

Forma de citar: García Delgado BM, Silva AP, de Rodríguez JM. Mapa conceptual en salud y propiedad intelectual en Centroamérica y la República Dominicana. Rev Panam Salud Publica. 2019;43:e4. https://doi. org/10.26633/RPSP.2019.4

RESUMEN Objetivo. El objetivo del presente estudio es elaborar un "mapa conceptual de salud pública y propiedad intelectual de Centroamérica y República Dominicana" que facilite la identificación de las principales barreras que inciden en el acceso a la salud, y en especial a los medicamentos, y que sirva de guía de acción a las autoridades estatales para mejorar la eficiencia de sus políticas y la efectividad de sus acciones.

Métodos. Se realizó el análisis del informe "MC SPPI; Perspectiva Regional: Centroamérica y República Dominicana". Posteriormente, se utilizó la Metodología para la Gestión y Generación de Conocimiento y se identificó la estrategia de búsqueda que permitió recobrar la información para la confección de los mapas conceptuales.

Resultados. Se identificaron el marco legal, la estructura institucional y las políticas, programas, planes y estrategias en temas de salud y propiedad intelectual, así como un conjunto de barreras que inciden negativamente en el acceso a los medicamentos y las soluciones que las contrarresten.

Conclusiones. En Centroamérica y la República Dominicana existen estrategias, políticas, programas y planes relacionados con la salud, aunque, lamentablemente, su alcance y grado de difusión difieren, lo que, unido a factores sociales y económicos, explica que persista la inequidad sanitaria entre los países. Entre las barreras al acceso a los medicamentos se identificaron la ampliación y refuerzo de los derechos de los titulares de patentes farmacéuticas, dificultades en la implementación de las flexibilidades de los Aspectos de los Derechos de Propiedad Intelectual relacionados con el Comercio, políticas de fijación de precios y estándares regulatorios estrictos.

Palabras clave Propiedad intelectual; legislación sanitaria; accesibilidad a los servicios de salud; derecho a la salud; Centroamérica; República Dominicana.

\footnotetext{
Fallecida el 12 de junio del 2018.

Consultora independiente. La Habana, Cuba. La correspondencia se debe dirigir a Beatriz M. García Delgado. Correo electrónico: beatrizgarcia@infomed.sld.cu

2 Sistemas y Servicios de Salud, Organización Panamericana de la Salud, Washington, DC; Estados Unidos de América.

3 Organización Panamericana de la Salud, Guatemala, Guatemala.
}

El derecho humano a la protección de la salud se ha reconocido en importantes documentos internacionales, como la Constitución de la Organización Mundial de la Salud (OMS, 1948), la Declaración de los Derechos Humanos (1949) y la Conferencia Internacional sobre Atención Primaria de Salud (1978).
Además, entre las metas de los Objetivos de Desarrollo del Milenio (ODM) se encuentra el acceso a los medicamentos. La OMS ha hecho hincapié en la necesidad de lograr la Cobertura Universal de Salud (CUS), cuyo objetivo es asegurar que todas las personas reciban los servicios sanitarios que necesitan (1). De 186

Este es un artículo de acceso abierto distribuido bajo los términos de la licencia Creative Commons Attribution-NonCommercial-NoDerivs 3.0 IGO, que permite su uso, distribución y reproducción en cualquier medio, siempre que el trabajo original se cite de la manera adecuada. No se permiten modificaciones a los artículos ni su uso comercial. Al reproducir un artículo no debe haber ningún indicio de que la OPS o el artículo avalan a una organización o un producto específico. El uso del logo de la OPS no está permitido. Esta leyenda debe conservarse, junto con la URL original del artículo. 
constituciones de países, 135 (73\%) incluyen provisiones para la salud o su cuidado $(2,3)$.

No obstante lo planteado, el acceso a la salud, a las tecnologías sanitarias y muy en especial a los medicamentos esenciales aún se encuentra alejado del alcance de la población en muchos países y a ello se suma la inequidad en el acceso entre las regiones y países y entre diferentes grupos poblacionales en un mismo país. Diversos autores han tratado diferentes aspectos (éticos, derechos humanos, económicos, entre otros) relacionados con el acceso a los medicamentos (4-6).

La elevación de los estándares de protección de las patentes farmacéuticas (o el aumento de su exigencia), que se establecieron a partir de la adopción del Acuerdo sobre Aspectos de los Derechos de Propiedad Intelectual relacionados con el Comercio (ADPIC), ha tenido una incidencia negativa en el acceso a los medicamentos. Según señala Velásquez (7), el tema de la propiedad intelectual, la salud pública y el acceso a medicamentos aparece mencionado por primera vez por la OMS en 1996, coincidiendo con la creación de la Organización Mundial del Comercio (OMC). Por otra parte, Correa analizó las posibles implicaciones del acuerdo sobre los ADPIC en el acceso a los medicamentos y el "margen de maniobra" que tiene el Acuerdo, para proteger la salud pública (8).

A lo anterior hay que añadir la suscripción de los países de Centroamérica y la República Dominicana a diferentes tratados y acuerdos comerciales internacionales (conocidos como ADPIC-PLUS), que han incidido en aspectos relacionados con las patentes farmacéuticas que presentan estándares de protección más fuertes o más exigentes que los definidos por los ADPIC.

El presente estudio parte de dos antecedentes. Primero, la Estrategia Global y Plan de Acción sobre Salud Pública, Innovación y Propiedad Intelectual (EGPA) contenida en la Resolución WHA61.21 de la OMS (9), dirigida a promover una innovación que tenga en cuenta las necesidades de acceso a las tecnologías sanitarias. Y segundo, la Perspectiva Regional sobre Salud Pública, Innovación y Propiedad Intelectual (PR-SPIPI) aprobada en la Resolución CD48.R15 de la Organización Panamericana de la Salud (OPS) (10), en la cual se insta a emprender acciones con el objetivo de comprometerse con la implementación a nivel nacional de la estrategia mundial y las partes acordadas en el plan de acción.

En este contexto, la OPS ha iniciado el Proyecto Mapa Conceptual sobre Salud Pública y Propiedad Intelectual (MC SPPI), dos de cuyos objetivos son compilar el marco legal en el ámbito de la gestión del derecho de propiedad intelectual (DPI) y salud pública que incide en el acceso a los medicamentos, y establecer un marco de análisis que ofrezca un diagnóstico para mejorar la eficiencia de sus programas y la efectividad de sus acciones en la promoción del acceso a tecnologías sanitarias en relación con la gestión de los DPI y sobre la base de los marcos legales y de las estructuras institucionales vigentes (11). Los resultados obtenidos al aplicar el MC SPPI a un caso de estudio muestran la utilidad que tiene elaborar el Mapa Conceptual que permitió identificar cómo la integración de su marco legal, la interrelación de los actores sociales y una voluntad política han influido favorablemente en el acceso de la población a la atención de la salud (12).

El objetivo de este estudio fue elaborar un "mapa conceptual de salud pública y propiedad intelectual de Centroamérica y la República Dominicana" que facilite identificar las principales barreras que obstaculizan el acceso a los servicios de salud, y en especial a los medicamentos, y que sirva de guía de acción a las autoridades estatales para mejorar la eficiencia de sus políticas y la efectividad de sus acciones.

\section{MATERIALES Y MÉTODOS}

El presente estudio partió del informe no publicado MC SPPI, Perspectiva Regional: Centroamérica y República Dominicana (2010 - 2014), en el cual se utilizó el cuestionario legal, administrativo y político-institucional desarrollado por la OPS, que se aplicó en 2010 en coordinación con las autoridades sanitarias y las Oficinas OPS de los países participantes de Centroamérica. En 2013, dicho informe se actualizó y se incluyó a la República Dominicana. Asimismo, se utilizó la Metodología para la Generación y Gestión del Conocimiento (MGGC), que está integrada por 6 etapas de las cuales se consideran factores críticos (FC) la 1, la 5 y la 6 (13-14). A continuación, se describen los objetivos de cada etapa:

- Etapa 1: Identificar y definir necesidades y problemática (FC). Revisión y análisis del referido informe y de los comentarios realizados por sus revisores e identificación de las necesidades de información para actualizar y dar respuestas a los comentarios de los revisores y a otros aspectos derivados de su análisis.

- Etapa 2: Planear la estrategia de búsqueda. Definición de resultados, indicadores y de la estrategia de búsqueda principal, de los procesos y las herramientas.

- Etapa 3: Recuperar información. Búsqueda y recuperación de información a partir de la Etapa 2.

- Etapa 4: Depurar y procesar información. Añadir valor.

En estas etapas se identificaron los sitios web y las bases de datos, se estableció el período de búsqueda y se seleccionaron las palabras clave. Con todo ello se recuperó la información que fue depurada y procesada. Se añadió valor e identificaron las necesidades de información que no habían sido recuperadas, se planeó una nueva estrategia de búsqueda (Etapa 2) y se procesó la información (Etapas 3 y 4).

- Etapa 5: Análisis y generación de conocimiento (FC). Se analizó la información recobrada, lo que permitió generar nuevo conocimiento a partir del cual, en 2016, se redactó el informe no publicado "Mapa conceptual en salud $y$ propiedad intelectual en Centroamérica y República Dominicana".

- Etapa 6: Difusión del conocimiento e inteligencia obtenidos (FC). Se difundió el conocimiento generado y se elaboró el presente manuscrito para su divulgación.

\section{RESULTADOS}

Se obtuvo información general sobre propiedad intelectual, el acceso a los servicios de salud y los tratados internacionales que inciden en el acceso a las tecnologías sanitarias. En los diferentes países participantes en este estudio se identificaron sus constituciones, leyes, decretos y reglamentos relacionados con los temas de propiedad intelectual, salud y medicamentos. Se identificaron actores y estructuras vinculados con la salud y la propiedad intelectual, así como los tratados internacionales suscritos, en especial el CAFTA-DR y el Tratado de 
Cooperación Comercial de Panamá con Estados Unidos.

Con el procesamiento, análisis y compilación de la referida información se construyeron los mapas conceptuales correspondientes a cada país. En los cuadros 1, 23 , se incluye un breve resumen de su contenido por país.

Por otro lado, se redactó el Informe "Proyecto: Mapa Conceptual en Salud Pública y Propiedad Intelectual. Perspectiva Regional: Centroamérica y República Dominicana", en el cual se exponen los resultados obtenidos para cada país. La información de este informe se estructuró en dos partes: 1) Marco Legal en Salud Pública, Estructura Institucional en
Salud Pública, y Política Nacional de Salud y Medicamentos, y 2) Marco Legal General en Propiedad Intelectual, Estructura Institucional de Propiedad Intelectual, Política Nacional de Propiedad Intelectual, Patentes y Flexibilidades. Seguidamente se presenta un breve resumen por país.

\section{Costa Rica}

El sector salud de Costa Rica está integrado por instituciones públicas encargadas de "proteger y mejorar el estado de salud de la población". Existen programas y planes relacionados con la salud, los medicamentos esenciales, la propiedad intelectual, así como con la ciencia, la innovación y la tecnología, entre los cuales destaca a Política Nacional de Salud, la base del Plan Nacional de Salud.

El Plan Nacional de Ciencia y Tecnología de la Salud plantea "que se busca consolidar un Sistema que articule los actores y acciones para que respondan de manera sinérgica a las necesidades de la población y que estimule el desarrollo de I+D+i que permita avances importantes en materia de salud".

El Plan Nacional de Ciencia, Tecnología e Innovación señala la importancia de incentivar la investigación interdisciplinar y a los investigadores en el

\section{CUADRO 1. Proyecto Mapa Conceptual sobre Salud Pública y Propiedad Intelectual. Mapa Conceptual de Salud Pública: Marco Legal y Actores}

\begin{tabular}{|c|c|c|}
\hline País & Mapa Conceptual Salud Pública: Marco Legal & Mapa Conceptual Salud Pública: Actores \\
\hline Costa Rica & $\begin{array}{l}\text { Constitución } \\
\text { https://www.ucr.ac.cr/medios/documentos/2015/constitucion_politica.pdf } \\
\text { Ley General de Salud } \\
\text { https://www.ucr.ac.cr/medios/documentos/2015/LEY-5395.pdf } \\
\text { Ley Orgánica del Ministerio de Salud Pública } \\
\text { https://www.pani.go.cr/publicaciones/documentos/leyes/259-ley-organica-del-ministerio-de- } \\
\text { salud-no-5412/file }\end{array}$ & $\begin{array}{l}\text { Ministerio de Salud } \\
\text { https://www.ministeriodesalud.go.cr/ } \\
\text { Sector Salud } \\
\text { https://www.mideplan.go.cr/360-catalogo-de- } \\
\text { cooperacion-tecnica/sector-salud } \\
\text { Sistema Nacional de Salud } \\
\text { http://www.binasss.sa.cr/revistas/hcr/n291994/art8.pdf } \\
\text { Caja Costarricense de Seguro Social } \\
\text { http://www.ccss.sa.cr/ } \\
\text { Colegio de Farmacéuticos de Costa Rica } \\
\text { http://www.colfar.com/index.php?option=com_revista } \\
\text { Universidades }\end{array}$ \\
\hline El Salvador & $\begin{array}{l}\text { Constitución https://www.oas.org/dil/esp/Constitucion_de_la_Republica_del_Salvador_1983.pdf } \\
\text { Ley de Creación del SNS http://asp.salud.gob.sv/regulacion/pdf/ley/Ley_sistema_nacional_salud.pdf } \\
\text { Ley de Medicamentos, https://www.defensoria.gob.sv/images/stories/descargas/Ley\%20de\%20 } \\
\text { Medicamentos.pdf } \\
\text { http://asp.salud.gob.sv/regulacion/pdf/reglamento/reglamento_ley_medicamentos.pdf }\end{array}$ & $\begin{array}{l}\text { Ministerio de Salud y Asistencia Social } \\
\text { http://www.salud.gob.sv/ } \\
\text { Dirección Nacional de Medicamentos http://www. } \\
\text { medicamentos.gob.sv/index.php/es/ }\end{array}$ \\
\hline Guatemala & $\begin{array}{l}\text { Constitución } \\
\text { https://www.oas.org/juridico/mla/sp/gtm/sp_gtm-int-text-const.pdf } \\
\text { Código de Salud http://asisehace.gt/media/GT_Codigo_Salud_90_97.pdf }\end{array}$ & $\begin{array}{l}\text { Ministerio de la Salud } \\
\text { http://www.mspas.gob.gt/ }\end{array}$ \\
\hline
\end{tabular}

$\begin{array}{ll}\text { Nicaragua } & \text { Constitución http://legislacion.asamblea.gob.ni/Normaweb. } \\ & \text { nsf/3133c0d121ea3897062568a1005e0f89/06c0db3b7bcfc75706257307006f6c6d? } \\ & \text { OpenDocument } \\ & \text { Ley General de la Salud https://extranet.who.int/nutrition/gina/sites/default/files/NIC\%20Ley\%20 } \\ & \text { 423\%20Ley\%20General\%20de\%20Salud.pdf } \\ & \text { Ley de Medicamentos http://www.vertic.org/media/National\%20Legislation/Nicaragua/NI_ } \\ & \text { Decreto_001-2003_Rgto_Ley_General_Salud.pdf } \\ \text { Panamá } & \text { Constitución http://www.asamblea.gob.pa/cep/const_constitucion1941.pdf } \\ & \text { Código Sanitario http://www.css.org.pa/CODIG0\%20SANITARIO\%20gaceta.pdf } \\ & \text { Ley sobre Medicamentos http://www.minsa.gob.pa/sites/default/files/publicacion-general/ } \\ & \text { ley-1-de-2001-sobre-medicamentos.pdf } \\ & \text { Estatuto Orgánico del Ministerio de Salud https://docs.panama.justia.com/federales/ } \\ & \text { decretos/75-de-1969-sep-2-1969.pdf }\end{array}$

República Constitución

Dominicana http://www.poderjudicial.gob.do/documentos/PDF/constitucion/Constitucion.pdf Ley General de Salud www.wipo.int/edocs/lexdocs/laws/es/do/do054es.pdf Reglamento de Medicamentos https://www.ictsd.org/sites/default/files/event/2009/08/rathe.ppt
Ministerio de Salud http://www.minsa.gob.ni/

\section{Ministerio de Salud}

https://www.minsa.gob.pa/

Dirección Nacional de Farmacia y Drogas https://www. minsa.gob.pa/direccion/direccion-nacional-de-farmacia-ydrogas

Autoridad Sanitaria Nacional

http://www.minsa.gob.pa/informacion-salud/registrosanitario-principal

Caja del Seguro Social

http://www.css.org.pa/

Ministerio de Salud y Asistencia Social

www.sespas.gov.do/

Seguro Nacional de Salud

http://www.arssenasa.gov.do/index/aboutsenasa.asp 
CUADRO 2. Proyecto Mapa Conceptual sobre Salud Pública y Propiedad Intelectual. Mapa Conceptual de Propiedad Intelectual: Marco Legal y Actores

\begin{tabular}{ll}
\hline País & Mapa Conceptual Propiedad Intelectual: Marco Legal \\
\hline Costa Rica & $\begin{array}{l}\text { Constitución https://www.ucr.ac.cr/medios/documentos/2015/constitucion_politica.pdf } \\
\text { Ley de Patentes y Modelos de Utilidad http://www.registronacional.go.cr/propiedad_industrial/ } \\
\text { documentos/pi_normativa/leyes/Ley\%20de\%20Patentes\%20y\%20su\%20Reglamento.pdf }\end{array}$
\end{tabular}

\begin{tabular}{|c|c|}
\hline Salvador & $\begin{array}{l}\text { Constitución https://www.oas.org/dil/esp/Constitucion_de_la_Republica_del_Salvador_1983.pdf } \\
\text { Ley de Patentes, y Modelos de Utilidad http://www.rnp.go.cr/propiedad_industrial/Documentos/ } \\
\text { Informacion\%20patentes\%20de\%20invencion,\%20modelos\%20de\%20utilidad\%20y\%20 } \\
\text { diseno\%20industrial.pdf } \\
\text { Ley de Creación del Registro Nacional https://www.asamblea.gob.sv/sites/default/files/documents/ } \\
\text { decretos/171117_072905898_archivo_documento_legislativo.pdf }\end{array}$ \\
\hline Guatemala & $\begin{array}{l}\text { Constitución } \\
\text { https://www.oas.org/juridico/mla/sp/gtm/sp_gtm-int-text-const.pdf } \\
\text { Ley de Propiedad Industrial y sus reglamentos http://www.wipo.int/edocs/lexdocs/laws/es/gt/ } \\
\text { gt001es.pdf }\end{array}$ \\
\hline Nicaragua & $\begin{array}{l}\text { Constitución } \\
\text { http://legislacion.asamblea.gob.ni/Normaweb. } \\
\text { nsf/3133c0d121ea3897062568a1005e0f89/06c0db3b7bcfc75706257307006f6c6d?0penDocument } \\
\text { Ley de patentes de invención, modelo de utilidad y diseños industriales http://www.tramitesnicaragua } \\
\text { gob.ni/media/Ley\%20354\%20Ley\%20de\%20Patentes\%20de\%20Invenci\%C3\%B3n,\%20Modelo\%2C } \\
\text { de\%20Utilidad\%20y\%20Dise\%C3\%B10s\%20Industriales.pdf }\end{array}$ \\
\hline Panamá & $\begin{array}{l}\text { Constitución } \\
\text { http://www.asamblea.gob.pa/cep/const_constitucion1941.pdf } \\
\text { Ley de propiedad industrial } \\
\text { http://www.legalinfo-panama.com/legislacion/propint/ley351996.pdf } \\
\text { Ley de patentes de invención, modelo de utilidad. http://www.wipo.int/edocs/lexdocs/laws/es/pa/ } \\
\text { pa038es.pdf }\end{array}$ \\
\hline $\begin{array}{l}\text { República } \\
\text { Dominicana }\end{array}$ & $\begin{array}{l}\text { Constitución } \\
\text { https://es.slideshare.net/cesrd/constitucion-republica-dominicana-2015 } \\
\text { Ley de propiedad Industrial } \\
\text { https://www.intec.edu.do/downloads/pdf/vriv/emprendurismo-innovacion/recursos/ley-20-00-en- } \\
\text { espanol.pdf }\end{array}$ \\
\hline
\end{tabular}

Mapa Conceptual Propiedad Intelectual: Actores

Ministerio de Justicia y Paz

http://www.mip.go.cr/

Registro Nacional

http://www.registronacional.go.cr/

Registro de la Propiedad Industrial http://www. registronacional.go.cr/propiedad_industrial/index.htm Universidades

Ministerio de Economía

http://www.minec.gob.sv/

Registro de la Propiedad Intelectual

http://www.cnr.gob.sv/introduccion-registro-de-lapropiedad-intelectual/

Ministerio de Economía

www.mineco.gob.gt/

Registro de la Propiedad Intelectual

https://rpi.gob.gt/

Registro de la Propiedad Intelectual https://www.mific. gob.ni/es-ni/registrodelapropiedadintelectual.aspx

Fiscalía Especializada en Delitos contra la PI http://

registronacional.go.cr/propiedad_industrial/

Documentos/PI\%20en\%20Nicaragua.pdf

Dirección del Registro de la Propiedad Intelectual http:// www. mici.gob.pa/subcategoria.php?cid= $16 \&$ sid $=52$

Ministerio de Comercio e Industrias

https://www.mici.gob.pa

Universidad Tecnológica de Panamá

http://www.utp.ac.pa/

Ministerio de Industria y Comercio

https://micm.gob.do/

Oficina Nacional de la Propiedad Industrial

www.onapi.gov.do/

Academia Nacional de la Propiedad Intelectual

www.onapi.gov.do/index.php/academia/academia

Procuraduría General de la República

https://pgr.gob.do/ campo de la salud. Además, existe un entramado legal de Propiedad Intelectual y una Estrategia Nacional de Propiedad Intelectual.

\section{El Salvador}

El Plan Estratégico Institucional de la Salud señala la necesidad de reconocer las diferencias y necesidades específicas de los diversos grupos poblacionales. Su objetivo principal es asegurar, progresivamente, el acceso y la cobertura universales en salud, haciendo especial hincapié en la calidad de atención que se presta a la población, en garantizar la salud como un derecho humano y en brindar sostenibilidad jurídica y financiera a la Reforma de Salud para fortalecer la rectoría, la integración y la gobernanza del Sistema Nacional de Salud. Dicho Plan plantea, asimismo, que la reforma innovadora y profunda del Sistema de Salud es un camino hacia la cobertura universal de salud, en el cual desempeñarán un papel importante la voluntad política del gobierno y el apoyo de la OPS.

\section{Guatemala}

La Política General de Gobierno (20162020) de este país señala que "una de las prioridades más importantes del Gobierno es la reforma del sector salud" y afirma, en primer lugar, que el Estado no es capaz de cumplir con el papel de garantizar el acceso universal a la salud ni de hacer frente, como ha quedado patente, al elevado desabastecimiento de medicamentos e insumos críticos en los hospitales públicos y en los centros y puestos de salud.

Se han elaborado planes que proponen el acceso a los servicios de salud, por ejemplo, el Plan de Desarrollo K’atun y el Proceso de la Reforma al Sector Salud en Guatemala, Saliendo de la Crisis y Construyendo el Futuro, que trazan políticas, estrategias y directrices encaminadas a solucionar la situación actual.

Estos problemas ponen de relieve la necesidad de capacitar y formar a los actores sociales, de contar con voluntad política y de difundir ampliamente lo plasmado en los referidos documentos para dar un vuelco a la situación descrita.

\section{Nicaragua}

La Política Nacional de Salud de este país se elaboró conforme al Plan Nacional de Desarrollo y las Metas del Milenio. El Ministerio de Salud se encarga del seguimiento de su implementación. Por otro lado, en el área de los medicamentos, se ha creado el Centro de Información de Medicina, que está orientado hacia la promoción de su uso apropiado.

Nicaragua fue la sede de la IV Reunión Ministerial Centroamericana sobre Propiedad Intelectual, cuyo objetivo 
CUADRO 3. Proyecto Mapa Conceptual sobre Salud Pública y Propiedad Intelectual. Mapas Conceptual de Salud Pública y de la Propiedad Intelectual: Políticas, planes y estrategias y programas nacionales

\begin{tabular}{|c|c|c|}
\hline País & Mapa Conceptual Salud Pública & Mapa Conceptual Propiedad Intelectual \\
\hline Costa Rica & $\begin{array}{l}\text { Política Nacional de Salud "Dr. Juan Guillermo Ortiz Guier" (2015-2018) } \\
\text { http://www.binasss.sa.cr/plannacionalsalud.pdf } \\
\text { Plan Nacional de Salud } \\
\text { http://www.binasss.sa.cr/plannacionalsalud.pdf } \\
\text { Plan Nacional de Salud y de Ciencia, Tecnología e Innovación https://www. } \\
\text { ministeriodesalud.go.cr/index.php/investigacion-y-tecnologia-en-salud/plan- } \\
\text { nacional-de-ciencia-tecnologia-en-salud/file } \\
\text { Plan Nacional de Ciencia, Tecnología e Innovación 2015-2021 http://pncti.micit. } \\
\text { go.cr/ }\end{array}$ & $\begin{array}{l}\text { Estrategia Nacional de Propiedad Intelectual (2012 } \\
\text { http://unpan1.un.org/intradoc/groups/public/documents/icap/ } \\
\text { unpan050424.pdf }\end{array}$ \\
\hline Guatemala & $\begin{array}{l}\text { Plan de Desarrollos K'atun, } 2032 \\
\text { http://www.segeplan.gob.gt/downloads/2015/SPOT/Mandatos_y_Normativas/ } \\
\text { Politicas/Politica_Nacional_de_Desarrollo.pdf } \\
\text { Proceso de la Reforma al Sector Salud http://www.mspas.gob.gt/images/files/ } \\
\text { procesoreforma/ProcesodelaReformaalSectorSaludenGuatemala.pdf }\end{array}$ & $\begin{array}{l}\text { Estrategia Nacional de Propiedad Intelectual http://www.wipo.int/edocs/ } \\
\text { mdocs/tk/es/wipo_iptk_ge_2_16/wipo_iptk_ge_2_16_ } \\
\text { presentation_14garcia.pdf }\end{array}$ \\
\hline Nicaragua & $\begin{array}{l}\text { Política Nacional de Salud } \\
\text { http://apps.who.int/medicinedocs/documents/s18995es/s18995es.pdf } \\
\text { Política de Medicamentos } \\
\text { https://www.paho.org/els/index.php?option=com_- } \\
\text { docman\&view=download\&category_slug=politicas-de-salud\&alias=811-politica- } \\
\text { nacional-de-medicamentos-18012012\&ltemid=364 } \\
\text { Plan Estratégico Nacional de Promoción del Uso Racional de Medicamentos } \\
\text { PENPURM (2011-2013) http://www.minsa.gob.ni/index.php/repository/Descargas- } \\
\text { MINSA/Divisi\%C3\%B3n-General-de-Insumos-M\%C3\%A9dicos/PENPURM-Plan- } \\
\text { Estrat\%C3\%A9gicos-Nacional-de-Promoci\%C3\%B3n/ } \\
\text { Plan Nacional de Ciencia, Tecnología e Innovación 2010-2013 http://docs. } \\
\text { politicascti.net/reportes/NI.pdf }\end{array}$ & $\begin{array}{l}\text { Ejes para un Programa Nacional de Desarrollo Humano (PNDH) } \\
\text { www.hacienda.gob.ni/hacienda/ppresupuesto2018/mpmp/204. } \\
\text { EjesProgramaNacionalDesarrolloHumano.pdf }\end{array}$ \\
\hline $\begin{array}{l}\text { República } \\
\text { Dominicana }\end{array}$ & $\begin{array}{l}\text { Política Farmacéutica Nacional } \\
\text { apps.who.int/medicinedocs/documents/s23004es/s23004es.pdf } \\
\text { Estrategia Nacional de Desarrollo } 2030 \\
\text { economia.gob.do/mepyd/estrategia-nacional-de-desarrollo-2030 }\end{array}$ & $\begin{array}{l}\text { Estrategia Nacional de la Propiedad Intelectual } \\
\text { www.onapi.gov.do/images/pdf/comunicaciones/estrategia_pi.pdf }\end{array}$ \\
\hline
\end{tabular}

principal fue abordar programas y acciones específicas para fortalecer la protección del intelecto en la subregión. En ella se acordó crear los Centros de Apoyo a la Tecnología e Innovación, una actividad que integra a las oficinas de propiedad intelectual y universidades, entre otras instituciones que disponen de Centros de
Apoyo a la Tecnología e Innovación nacionales en la región.

\section{Panamá}

Panamá posee una Política Nacional de Salud y su Política Nacional de Medicamentos aborda aspectos relacionados con la propiedad intelectual. Según dicha política, "Las autoridades sanitarias panameñas reconocen la creciente importancia que tienen las medidas de protección de la propiedad intelectual sobre los medicamentos en relación con la contención de costos y la mejora de las condiciones de acceso y cobertura sanitaria. 


\section{CUADRO 4. Barreras al acceso a las tecnologías sanitarias y medicamentos}

Ampliación y refuerzo de los derechos de los titulares de patentes farmacéuticas a partir de la adhesión de los
países de la región a IOS ADPIC y a los ADPIC+PLUS, lo que dificulta aún más la implementación de las
flexibilidades de los ADPIC y de la Ronda de Doha
Dificultades en la implementación de las flexibilidades y dificultades para el uso de las que se han implementado
en las leyes
Concesión de derechos a titulares de patentes espurias, estrategias "evergreening"
Políticas de fijación de precios
Limitadas capacidades científicas-tecnológicas y de recursos humanos para el desarrollo de medicamentos
Limitada presencia a nivel mundial de investigación y desarrollo de medicamentos y vacunas para enfermedades
prevalentes en los países en desarrollo
Incremento constante de los estándares regulatorios

Las autoridades sanitarias consideran necesario recomendar medidas que favorezcan la gestión de los medicamentos.

Por último, la Universidad Tecnológica de Panamá participa activamente en la formación de capital humano en temas de propiedad intelectual a través del Centro de Propiedad Intelectual.

\section{República Dominicana}

Este país tiene programas y planes relacionados con la salud, los medicamentos esenciales, la propiedad Intelectual, así como con la ciencia, la innovación y la tecnología.

Entre las líneas de acción de la Estrategia Nacional de Desarrollo 2030 destaca la que persigue el objetivo de "Garantizar a toda la población, el acceso a medicamentos de calidad, promover su uso racional y priorizar la disponibilidad de medicamentos de fuentes múltiples (genéricos) en el mercado nacional".

Por su parte, la Estrategia Nacional de la Propiedad Intelectual es el producto un plan piloto enmarcado en el Proyecto de la Agenda para el Desarrollo de la OMPI, con el cual se pretende integrar a la Propiedad Intelectual en las políticas públicas.

\section{DISCUSIÓN}

Los países objeto de estudio poseen un marco legal, programas, planes y estrategias vinculados con la salud y la propiedad intelectual cuyo alcance de forma general difiere entre ellos. Las legislaciones se han adecuado a las exigencias de los acuerdos suscritos internacionalmente (ADPIC, CAFTA-DR, Acuerdo de Promoción Comercial), así como al alcance de lo suscrito por cada país en dichos acuerdos.
En la bibliografía se ha reflejado el impacto que han tenido los ADPIC y los ADPIC PLUS relacionados con la propiedad intelectual en el precio de los medicamentos (15-16), así como otros aspectos asociados con la incidencia de los ADPIC PLUS en lo referente a las esferas de la economía y la salud (17-19). Gobiernos y organizaciones internacionales le prestan especial atención a este problema analizando las diversas soluciones que pueden minimizar esta situación. Uno de los aspectos más estudiados es la incorporación de las flexibilidades de los ADPIC, en las legislaciones nacionales.

Se ha señalado que las disposiciones que prolongan la duración de la patente más allá de los veinte años que exige la Organización Mundial del Comercio (OMC) y aquellas que disminuyen los criterios sobre lo que puede patentarse y las disposiciones sobre la exclusividad de los datos introducen trabas a los productores de medicamentos genéricos que entran en los mercados tras la expiración de las patentes.

El informe elaborado por el grupo de Alto Nivel del Secretario General de las Naciones Unidas sobre el acceso a los medicamentos refleja claramente los problemas existentes relacionados con el uso de las flexibilidades (20). En él se indica que las flexibilidades en materia de salud pública en el marco legal de propiedad intelectual competen a diversas entidades gubernamentales, como el comercio, la ciencia y la tecnología, la industria y las finanzas, entre otros.

Los países estudiados (excepto Panamá, que es signatario del Tratado de Promoción Comercial entre Panamá y Estados Unidos) son signatarios del CAFTA-DR y ello ha influido en la aplicación de las flexibilidades de los ADPIC e incide negativamente en la entrada en el mercado de medicamentos genéricos $\mathrm{y}$, por consiguiente, en el acceso a servicios de salud.

Entre los aspectos que limitan el uso de las referidas flexibilidades se encuentran los siguientes: importaciones paralelas, criterios de patentabilidad, excepciones generales, concesión de licencias obligatorias, uso por el gobierno, disposiciones relacionadas con la competencia y periodos transitorios. Las flexibilidades, aunque estén debidamente adecuadas en las legislaciones nacionales, pueden no ser efectivas por diferentes motivos, algunos intrínsecos a los países y otros que se ven afectados por el entorno internacional.

En el caso de los países que han suscrito tratados de libre comercio, como el CAFTA-DR, la situación es más complicada. Lo ejemplifica, por ejemplo, el régimen sui generis de protección de los datos de prueba presentados para el registro de productos farmacéuticos, que implica estándares más elevados o exigentes que los requeridos por los ADPIC (21), al igual que las "ampliaciones de los plazos de las patentes por demoras reglamentarias o comerciales poco razonables y la disposición relativa a la "vinculación de patentes" (22).

Los resultados obtenidos indican que es necesario propiciar una mayor interacción entre los diferentes actores sociales e identificar algunas de las barreras que reducen el acceso a las tecnologías sanitarias y en especial a los medicamentos (cuadro 3).

Los altos precios de los medicamentos afectan tanto a países desarrollados como en desarrollo y son una de las mayores barreras al acceso a los medicamentos, que depende, además, de otros factores, como la imposibilidad de acceder a medicamentos genéricos a precios mucho más bajos.

Se barajan soluciones alternativas que pueden mejorar el acceso a los medicamentos, como las que se enumeran a continuación:

- Desarrollo de políticas farmacéuticas nacionales y de la subregión objeto de este estudio e implementación de políticas de desarrollo y producción de medicamentos genéricos.

- Elaboración de normas de examen de patentes que sean muy rigurosas y refuerzo de las medidas para su cumplimiento. 
- Divulgación y utilización de la información relacionada con las patentes farmacéuticas concedidas y solicitadas y utilización de las bases de datos de patentes y de las de uso de la información contenida en los documentos de patentes en los procesos de investigación, desarrollo e innovación.

- Capacitación de los recursos humanos en temas de propiedad intelectual que puedan incidir positivamente en la implementación de las flexibilidades.

- Capacitación de examinadores de patentes para que sólo se otorguen derechos a las patentes que cumplan los requisitos exigidos, velando para que cada patente que se conceda tenga un verdadero valor inventivo y evitando la concesión de patentes espurias.

- Creación de capacidades en recursos humanos, en investigación y desarrollo y de capacidades productivas para la fabricación de medicamentos.

- Utilización de los mecanismos de compra conjunta. En la actualidad, los países de la Región de las Américas tienen acceso a dos mecanismos de compra conjunta: la Negociación Conjunta y Compra de Medicamentos (Negociación Conjunta COMISCA) y el Fondo Estratégico de la OPS, que es un mecanismo solidario de compras conjuntas de medicamentos esenciales y suministros de salud estratégicos para todos los países de la Región.

- Aplicación de las licencias obligatorias y otras flexibilidades.

Se puede afirmar que, en general, en los países estudiados existen estrategias, políticas, programas y planes relacionados con la salud, aunque, lamentablemente, su alcance y difusión son diferentes, lo que, unido a factores sociales y económicos y en ocasiones a una limitada voluntad política, explica que persista la inequidad sanitaria entre los países de la Región y en cada uno de ellos. A este respecto se ha reiterado que “en la Región persisten otras inequidades considerables en torno a la salud como consecuencia de la exclusión social y cultural, el desempleo, las condiciones de trabajo deficientes, la falta de educación e información, el aislamiento geográfico, los entornos poco saludables y otros determinantes sociales" (23).

Los análisis comparativos realizados de los programas, planes o estrategias nacionales (en salud y en propiedad intelectual) muestran, primero, que en algunos países se necesita actualizar sus objetivos y adecuarlos a la situación vigente a nivel mundial y de los países y, segundo, que se deben identificar los retos existentes y las mejores soluciones para resolverlos en el menor tiempo posible. Asimismo, es necesario contar con la voluntad política de los Estados para afrontar los retos y las barreras que limitan el acceso a los medicamentos.

Este artículo es un resumen de lo expuesto en el Informe "Mapa Conceptual en Salud Pública y Propiedad Intelectual. Perspectiva Regional: Centroamérica y República Dominicana" y, por ello, no aparecen todos los resultados y elementos que se exponen en él, lo cual justifica que se realice una nueva publicación en la cual se analicen en detalle los resultados de algunos de los países estudiados.

Cabe concluir que en la Región existen estrategias, políticas, programas y planes relacionados con la salud, aunque su alcance y difusión difieren. Esto, unido a factores sociales y económicos explica que persista la inequidad sanitaria entre los países de la Región. Entre las barreras al acceso a los medicamentos se han identificado la ampliación y el refuerzo de los derechos de los titulares de patentes farmacéuticas, dificultades en la implementación de las flexibilidades de los ADPIC, políticas de fijación de precios y estándares regulatorios estrictos.

Los marcos legales de salud y propiedad intelectual en vigor no dan respuesta en todos los casos a los retos ni a las barreras identificados para lograr el acceso a los servicios de salud y especialmente a los medicamentos. Entre ellos se encuentran los relacionados con las flexibilizaciones de los ADPIC. Además, los programas, planes o estrategias de salud, aprobados en algunos casos, no se han actualizado conforme a la situación vigente respecto al acceso a los servicios de salud y a los medicamentos. Por otro lado, es preciso adecuar las legislaciones para que se puedan utilizar las flexibilizaciones de los ADPIC.

La falta de rigor en el examen de las patentes farmacéuticas menoscaba el acceso a los medicamentos, toda vez que se conceden derechos a productos que no cumplen con el carácter innovador, lo cual alarga los derechos de patente y limita la entrada de productos genéricos a precios más bajos. Debe hacerse hincapié en que el acceso limitado a los medicamentos es un problema que afecta a la población mundial y que en él inciden múltiples factores.

Para superar las barreras existentes es necesario partir de las debilidades y amenazas derivadas de los tratados suscritos, de la situación de la economía global y de la economía en la Región estudiada y del cambio climático, así como tener en cuenta las siguientes recomendaciones.

Primero, perfeccionar los programas y planes existentes, analizar profundamente la legislación actual y adecuarlos en aspectos relacionados, por ejemplo, con las licencias obligatorias y las importaciones paralelas.

Segundo, formar capital humano vinculado en el área del desarrollo y la innovación de las tecnologías sanitarias e incorporar en sus conocimientos aspectos básicos relacionados con la propiedad intelectual $\mathrm{y}$, en concreto, con la propiedad industrial, para que puedan hacer frente a los retos actuales.

$\mathrm{Y}$, para finalizar, formar recursos humanos en el uso de la información contenida en los documentos de patentes y capacitar a los examinadores de invenciones.

Agradecimientos. Las autoras desean expresar su agradecimiento a las autoridades sanitarias, de propiedad industrial y otras entidades de algunos de los países que forman parte de este estudio y que brindaron información para su realización, así como al Programa Subregional de Medicamentos de Centroamérica y República Dominicana de la OPS. Desean hacer una mención especial a la Dra. Ana Paula Silva, coautora de este trabajo, que lamentablemente falleció antes de la publicación de este artículo.

Financiación. Este estudio ha recibido financiación del Programa Subregional de Medicamentos de Centroamérica y República Dominicana de OPS.

Conflictos de interés. Las autoras declaran no tener conflictos de interés.

Declaración. Las opiniones expresadas en este manuscrito son responsabilidad de las autoras y no reflejan necesariamente los criterios ni la política de la RPSP/PAJPH y/o de la OPS. 


\section{REFERENCIAS}

1. Organización Mundial de la Salud. Cobertura Sanitaria Universal. Ginebra: OMS; 2012. Disponible en: http://www. who.int/universal_health_coverage/es/ Acceso el 10 julio de 2017.

2. Perehudoff SK, Laing RO, Hogerzeil HV. Access to essential medications in national constitutions. Bull WHO. 2010;1; 88(11):800.

3. Perehudoff SK. Health, essential medicines, human rights and national constitutions. Amsterdam: Vrije Universiteit Amsterdam; 2008. Disponible en: http:/ / www.who.int/medicines/areas/human_rights/Perehudoff_report_constitutions_2008.pdf Acceso el 16 de octubre de 2018.

4. Lage A. Desarrollo Farmacéutico Global y Acceso a Medicamentos: Temas Críticos de Ética y Equidad. MEDICC Review. 2011;13(3):1-8.

5. Hogerzeil H. Essential medicines and human rights: what can they learn from each other? Bull WHO. 2006;84(5):371-5.

6. Mirza Z, Krattiger A, Taubman A, Bartels $\mathrm{H}$, Beyer P, Kampf R, et al. Policy coherence for improved medical innovation and access. Bull WHO. 2013;91(5):315-315A. Disponible en: http://apps.who.int/me: dicinedocs/pdf/h2998e/h2998e.pdf Acceso el 27 de septiembre de 2017.

7. Velásquez G. El tema de la propiedad intelectual, la salud pública y el acceso a medicamentos en las organizaciones internacionales. Documento de investigación 78. Ginebra: Centro del Sur; 2017. Disponible en: https://www.southcentre. int/wp-content/uploads / 2017 / 07 / RP78_IP-Public-Health-and-Access-toMedicines-in-Internationa Organizations_ES.pdf Acceso el 25 de septiembre de 2017.

8. Correa CM. The Uruguay Round and drugs. Geneva: WHO Task Force on Health Economics, WHO Action Programme on Essential Drugs; 1997. Disponible en: http://apps.who.int/met dicinedocs/pdf/h2998e/h2998e.pdf Acceso el 25 de septiembre de 2017.

9. Organización Mundial de la Salud. Estrategia mundial y plan de acción sobre salud pública. Innovación y propiedad intelectual. Ginebra: OMS; 2008. Disponible en: http://apps.who.int/gb/CEWG/ pdf/A61_R21-sp.pdf Acceso el 11 julio de 2017.

10. Organización Panamericana de la Salud. Salud Pública, Innovación y Propiedad Intelectual: Una Perspectiva Regional.
Resolución CD48.R15. Washington, DC: OPS; 2015. Disponible en: http://www1. paho.org/hq/dmdocuments / $2010 / \mathrm{cd} 48$. r15-spanish.pdf Acceso el 11 julio de 2017

11. Vidal J. Salud Pública, Innovación y Propiedad Intelectual en las Américas. Reunión Regional de Consulta "Integración y Convergencia para la Salud en América Latina y el Caribe". Caracas: SELA; 2010. Disponible en: http://www. sela.org/media/266299/t023600004267-0di_13_jaume_vidal-ops_reunion_regional_salud.pdf Acceso el 10 de agosto de 2017.

12. García B, Di Fabio J, Vidal J, Fitzgerald J, Silva A. Salud pública y propiedad intelectual en Cuba: mapa conceptual. Rev Panam Salud Publica. 2015;38(5):355-61. Disponible en: http://iris.paho.org/ $\mathrm{x} \mathrm{m} \mathrm{lu} \mathrm{i} \mathrm{/} \mathrm{bit} \mathrm{s} \mathrm{t} \mathrm{r} \mathrm{e} \mathrm{a} \mathrm{m} \mathrm{/} \mathrm{h} \mathrm{a} \mathrm{n} \mathrm{d} \mathrm{-}$ le/123456789/18393/v38n5a2_355-361. pdf?sequence $=1 \&$ is Allowed $=y$ Acceso el 15 julio de 2017.

13. García B. Gestión y generación de conocimientos a partir de la información de patentes. Metodología. [Tesis de máster.] La Habana: Oficina Cubana de la Propiedad Industrial; 2012.

14. García B, Delgado M, Infante A. Metodología para la generación y gestión del conocimiento para proyectos de $\mathrm{I}+\mathrm{D}+\mathrm{i}$ a partir de sus factores críticos. Rev Cubana Información Ciencias Salud. 2014;25(3):285-302. Disponible en: ht1 tps: / / www.researchgate.net/publication/323015269_Gestion_y_Generacion_ de_Conocimientos_a_partir_de_la Informacion_de_Patentes_Metodologia Acceso el 28 de julio de 2017.

15. Correa C. Flexibilities provided by the Agreement on Trade-Related Aspects of Intellectual Property Rights. Bull WHO. 2018;96:148.

16. McNeill D, Birkbeck CD, Fukuda-Parr S, Grover A, Schrecker T, Stuckler D. Political origins of health inequities: trade and investment agreements. Lancet. 2017;389(10070):7602.

17. Organización Mundial de la Propiedad Intelectual. Significado de las flexibilidades. 2010. Disponible en. http://www. wipo.int/ip-development/es/agenda/ flexibilities/meaning_of_flexibilities.html Acceso el 28 de julio de 2017.

18. Organización Mundial de la Propiedad Intelectual. CDIP/5/4 Rev. - Flexibilidades en materia de patentes en el marco jurídico multilateral y su aplicación legislativa en los planos nacional y regional. Ginebra:
OMPI; 2010. Disponible en: http://www. wipo.int/meetings/es/doc_details.jsp?doc_id=153559 Acceso el 28 de julio de 2017.

19. Hernández G, Valverde H. Evaluación del Impacto de las Disposiciones de ADPIC + en el Mercado Institucional de Medicamentos de Costa Rica. Documento de Fondo No. 26. Ginebra: Centro Internacional para el Comercio y el Desarrollo Sostenible; 2009. Disponible en: https://www.ictsd.org/siw tes/default/files/research/2011/12/evaluacion-del-impacto-de-las-disposiciones-de-adpic-en-el-mercado-institucional-de-medicamentos-de-costa-rica.pdf Acceso el 10 de julio de 2017.

20.'t Hoen EFM, Veraldi J, Toebes B, Hogerzeil HV. The poorly understood power of TRIPS flexibilities: a comprehensive overview of their use in the procurement and supply of medicines 2001-2016. Bull WHO. 2018;96(3):185-93.

21. Naciones Unidas. Informe del Grupo de Alto Nivel del Secretario General de las Naciones Unidas sobre el acceso a los medicamentos. Promover la innovación y el acceso a las tecnologías de la salud. Nueva York: Naciones Unidas; 2016. Disponible en: https://static1.squarespace.com/ static/562094dee4b0d00c1a3ef761/ $\mathrm{t} / 596 \mathrm{feefb} 3$ e $00 \mathrm{be} 55 \mathrm{~b} 028 \mathrm{a} 1 \mathrm{a} 6 /$ 1500507901944/50923+-+HLP+Report_ SPANISH-v5_web.pdf Acceso el 16 de octubre de 2018.

22. Correa C. Implementación de la Protección de Datos de Prueba de Productos Farmacéuticos y Agroquímicos en DRCAFTA-ley Modelo. Ginebra: Centro Internacional para el Comercio y el Desarrollo Sostenible; 2006. Disponible en: https: / / www.ictsd.org / downloads /2009/06/7_ictsd20cafta20proteccion20de20datos_carlos_camerica.pdf Acceso 10 de julio de 2017.

23. Organización Panamericana de la Salud. Informe Anual. Washington, DC: OPS; 2016. Disponible en: http://www.paho. org/annual-report-2016/Espanol.html Acceso el 23 de septiembre de 2017.

Manuscrito recibido el 17 de octubre de 2017. Aceptado para publicación, tras revisión, el 10 de septiembre de 2018. 
ABSTRACT

\section{Concept Map on Health and Intellectual Property in Central America and the Dominican Republic}

Keywords
Objective. The objective of this study is to prepare a "Concept Map on Health and Intellectual Property in Central America and the Dominican Republic" in order to help identify the main access barriers to health, especially medicines, and to guide state authorities toward actions to improve the efficiency and effectiveness of policies.

Methods. The report "Concept Map on Public Health and Intellectual Property, Regional Perspective: Central America and the Dominican Republic" was analyzed. Then the knowledge generation and management methodology was applied and a search strategy was identified to recover the information for preparation of the concept maps.

Results. The legal framework and institutional structure, as well as policies, programs, plans, and strategies for health and intellectual property, were identified. A set of barriers that hinder access to medicines was also identified, as well as solutions to overcome them.

Conclusions. In Central America and the Dominican Republic, health strategies, policies, programs, and plans are in place, but unfortunately their scope and degree of dissemination is uneven. This, together with social and economic factors, explains why health inequity persists between and within countries. The identified barriers to access to medicines included the expansion and strengthening of the rights of pharmaceutical patent-holders, problems with implementing the flexibilities of the Agreement on Trade-related Aspects of Intellectual Property Rights, pricing policies, and strict regulatory standards.

Intellectual property; health legislation; health services accessibility; right to health; Central America; Dominican Republic.

RESUMO Objetivo. Elaborar um mapa conceitual de saúde pública e propriedade intelectual na América Central e na República Dominicana para facilitar identificar as principais barreiras ao acesso à saúde, em particular ao acesso aos medicamentos, e servir de guia às autoridades públicas para melhorar a eficiência das políticas e a efetividade das ações.

Mapa conceitual de saúde e propriedade intelectual na América Central e na República Dominicana

Métodos. Foi analisado o relatório Mapa conceitual de saúde pública e propriedade intelectual (MC SPPI); perspectiva regional: América Central e República Dominicana. Foi usada a metodologia para análise de gestão e geração de conhecimento e identificada a estratégia de busca que permitiu recuperar a informação para a construção dos mapas conceituais.

Resultados. Foram identificados o enquadramento jurídico, a estrutura institucional e as políticas, programas, planos e estratégias de saúde e propriedade intelectual e uma série de barreiras que interferem negativamente no acesso aos medicamentos e as soluções para eliminá-las.

Conclusões. Na América Central e na República Dominicana, existem estratégias, políticas, programas e planos de saúde, porém eles têm alcance e disseminação distintos que, ao lado de fatores sociais e econômicos, explicam por que persiste a falta de equidade em saúde entre os países. Entre as barreiras ao acesso aos medicamentos estão o reforço e a ampliação dos direitos dos detentores de patentes farmacêuticas, dificuldades na implementação das flexibilidades dos Aspectos dos Direitos de Propriedade Intelectual Relacionados ao Comércio, políticas de fixação de preços e normas reguladoras estritas.

Palavras-chave
Propriedade intelectual; legislação sanitária; acesso aos serviços de saúde; direito à saúde; América Central; República Dominicana. 